\title{
Stability of an Idealised Equilibrium Model of Prominences in Rapidly-Rotating Stars
}

Miguel Ferreira

School of Physics and Astronomy, University of St. Andrews, St. Andrews, Fife KY16 9SS, Scotland

Abstract. We study the stability of an idealised model of stellar prominences. We find that surface fields of a few hundred gauss are required to hold them in a stable equilibrium.

\section{Physical Considerations}

Prominence-like clouds have now been observed in many stars other than the Sun. These stars are invariably very rapid rotators and exhibit a strong magnetic activity. In contrast to their solar counterparts, there is a class of stellar prominences that do not lie close to the surface but rather at heights of several stellar radii. In particular, they are inferred to form close to, or beyond, the corotation radius $\left(R_{k}\right)$. This is the point at which the centrifugal force and gravity are in balanc e at the equatorial plane in a co-rotating atmosphere.

Let us first consider the simple problem of determining the locations where material could, in principle, accumulate to form a prominence in a prescribed magnetic field. This is illustrated in Figures 1 and 2 where we calculate the points at which the combine d effects of gravity and centrifugal force (that we denote as effective gravity, $\left.g_{\text {eff }}=-G M_{*} / r^{2}+\omega^{2} r\right)$ are in a stable balance along the direction of the field.
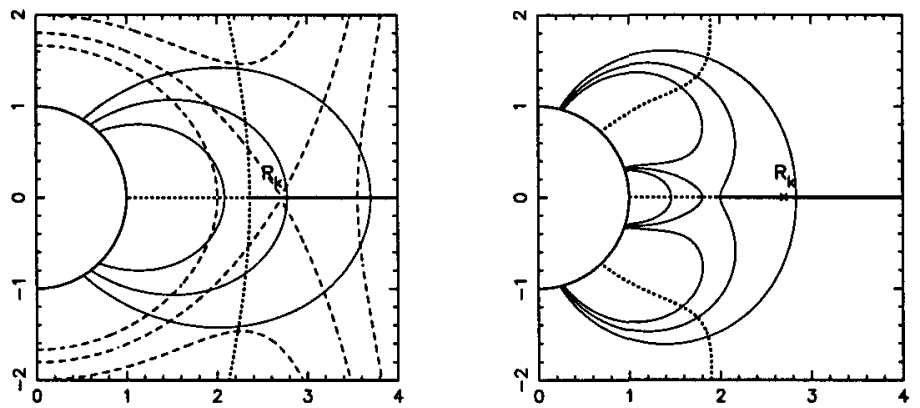

Figure 1. Equilibrium points for a dipole and a combined dipole and sextupole fields. Shown are the equipotential lines (dashed), field lines (light full), the unstable equilibrium points (dotted) and the stable equilibrium points (thick full). 


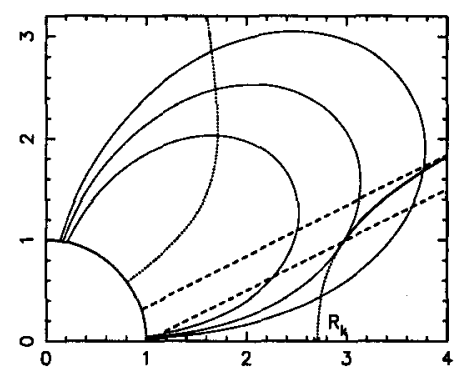

Figure 2. Equilibrium points for a quadrupolar field. The parallel dashed lines contain the part of stellar surface occulted by a prominence formed in the stable equilibrium region, as seen by a distant observer along that direction.

From these simple examples we infer that stable equilibrium along the direction of the field is possible with simple potential fields. Not so obvious is the fact that this can occur below $R_{k}$ even in the absence of a dipped configuration (Figure $1 \mathrm{a}$ ), while in other cases stable equilibrium is only possible further way from $R_{k}$ (Figure 2). Assuming that prominences form on some of these equilibrium surfaces, then, with the exception of the field configurations symmetric with respect to the stellar equator, they will in general have a curved shape.

If we make the analogy with solar prominences, we expect these stellar clouds to be thin structures along the direction of $\mathbf{B}$. If so, how can they be observed? This crucial question was raised during this conference by U. Anzer. Naturally, one can not rule out the possibility that stellar prominences are very different from their solar counterparts (e.g., Could they be evolving, cool postflare loops?). Nevertheless, if we assume this analogy, the question can be answered by the fact that the area of the prominence projected on the stellar surface along the direction of the observer is a substantial fraction of the total stellar surface area, therefore absorbing a significant fraction of the chromospheric flux (Figure 2). Also, noticing that in the region where stellar prominences form the pressure scale-height is about one order of magnitude larger than the solar value, we predict that stellar promine nces have a greater width than solar ones.

Let us now turn our attention to the equilibrium and stability of the prominence material in the direction perpendicular to the field. The ratio of the gas pressure gradient force to the effective gravity in the radial direction is

$$
\frac{\frac{d P}{d r}}{\rho g_{e f f}(r)} \approx \frac{p}{l_{0} \rho g_{e f f}}=\frac{R T_{p}}{l_{0} g_{e f f}}
$$

where $l_{0}$ is the typical radial extent of the prominence and $T_{p}$ its temperature. At the corotation radius $g_{e f f}=0$ and pressure is the only non-magnetic force present. However, if prominence material forms even a fraction of a stellar radius away from $R_{k}$ and for typical parameters $\left(T_{p}=10^{4} \mathrm{~K}, l_{0} \approx 10^{8}-10^{9} \mathrm{~m}\right)$, effective gravity dominates over pressure gradient and the above ratio is much smaller than one. We then neglect the gas pressure gradient force in the equi- 
librium equation and only look at the balance between the Lorentz force and effective gravity. It is reasonable to consider that stable equilibrium can occur in the prominence if the local magnetic energy is greater than the generalized gravitational potential energy:

$$
\frac{B^{2}}{2 \mu} \geq \rho\left[\frac{G M_{\star}}{r^{\prime}}+\frac{\omega^{2} r^{\prime 2}}{2}\right]_{r^{\prime}=R_{k}}^{r^{\prime}=r_{p}} .
$$

If the prominence lies sufficiently away from $R_{k}$ this condition is similar to imposing $v_{A} \geq \omega r_{p}$. Writing the unknown prominence mass density in terms of the mass density of solar prominences, $\rho=\left(\rho / \rho_{\odot}\right) \rho_{\odot}$, and taking $\rho_{\odot}=$ $1.6 \times 10^{-13} \mathrm{~g} \mathrm{~cm}^{-3}$, we obtain a minimum field strength, $B\left(r_{p}\right)$

$$
B(3)=5\left(\rho / \rho_{\odot}\right)^{1 / 2} \mathrm{G} ; \quad B(4)=20\left(\rho / \rho_{\odot}\right)^{1 / 2} \mathrm{G},
$$

which is similar to the field strength measured for solar prominences. Assuming a dipolar field one obtains a surface field of $B_{s}\left(r_{p}=3\right)=135 \mathrm{G}$ and $B_{s}\left(r_{p}=\right.$ 4) $=1.3 \mathrm{kG}$, respectively.

\section{Axisymmetric Massive Current Sheet Model}

In this model prominences are represented by an equatorial axisymmetric massive current sheet supported by a dipole field. After prescribing the surface current distribution, $\sigma(r) \equiv-\left[B_{r}\right] / \mu=-2 B_{r}^{+} / \mu$, the total magnetic field is computed by assuming that either the field is closed everywhere or it opens up at a given radius in order to simulate the effect of a wind (Figure 3 ). The surface mass density is then determined using the equilibrium equation, $m=-2 B_{r}^{+} B_{\theta} / \mu g_{\text {eff }}$.
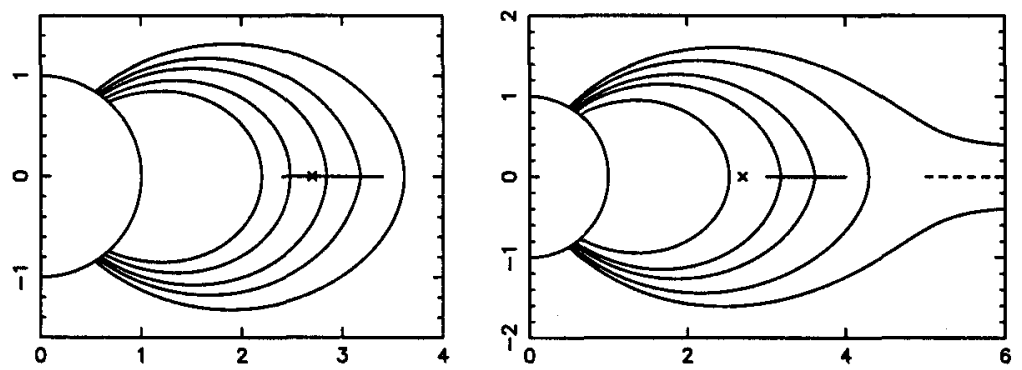

Figure 3. Prominences as massive current sheets. Left: The prominence extends from $r=2.4$ to $r=3.4$ and the field is closed everywhere. Right: The prominence extends from $r=3.0$ to $r=4.0$ and the field opens up at $r=5.0$. The represented configurations are stable.

In a rotating system there is no energy principle because of the Coriolis force. Nevertheless, by simply substituting gravity for $g_{\text {eff }}$ and neglecting the Coriolis force one can still apply the energy method of Bernstein et al. (1958) to obtain sufficient, though not necessary, linear and ideal stability conditions:

$$
-B_{r}^{+} \frac{\partial\left(r B_{\theta}\right)}{\partial r}-m \frac{\omega^{2}}{r} \leq+C
$$




$$
\frac{\partial B_{r}^{+}}{\partial r}-\frac{1}{g_{e f f}} \frac{\partial g_{e f f}}{\partial r} B_{r}^{+} \geq-A \Leftrightarrow \frac{\partial}{\partial r}\left(\frac{m}{\left|B_{\theta}\right|}\right) g_{e f f} \geq-A .
$$

The functions $A$ and $C$ represent the stabilizing effect of line-tying (Lepeltier and Aly 1996) and are such that $A, C \geq 0$. The above conditions are written assuming $B_{\theta} \leq 0, B_{r}^{+}>0\left(r>R_{k}\right)$. These stability criteria are similar to the ones derived for solar prominences (Anzer 1969). Condition (4) is related with the field curvature and is easily obeyed by the equilibrium models. The term $m \omega^{2} / r$ is stabilizing and allows for stable equilibrium to be possible for $r<R_{k}$ without the presence of a dip at the loop top (Figure 1a). Condition (5) states that the mass per unit flux cannot decrease outwards very fast and this can be associated with the magnetic Rayleigh-Taylor instability. While solar promin ences are most unstable to the interchange mode at their lower end, these stellar counterparts are most unstable at their upper end where the magnetic field strength is weaker and therefore less capable of stabilizing the equilibrium. A normal mode analysis restricted to short wavelength perturbations yields necessary stability conditions (Spruit and Taam 1990). In the particular case considered, with the equilibrium at the equatorial plane, conditions (4) and (5) are found to be both necessary and sufficient.

Masses of stellar prominences have been estimated to be of the order of $2-6 \times 10^{17} \mathrm{~g}$ (Collier-Cameron et al. 1990). Although the model is axisymmetric, we consider that the prominence mass is concentrated in a region of dimension $L$ in the azimuthal direction. We find that the minimum surface field strength necessary for a stable equilibrium is given by

$$
B_{s}=B_{0}\left(\frac{M_{p}}{5 \times 10^{17} g}\right)^{1 / 2}\left(\frac{r_{p}}{4 L}\right)^{1 / 2} \mathrm{G},
$$

where $B_{0}$ depends on the following: the prominence location $\left(r_{p}\right)$, its radial extent, the mass distribution profile along it and also how close the prominence is to the open field region. Making sensible choices one obtains $B_{0}=200-800 \mathrm{G}$. The kinetic energy of these prominences is of the order of $10^{32}-10^{33} \mathrm{erg}$ while the free magnetic energy calculated using stable equilibrium models is almost one order of magnitude lower. The energy of the flares observed on these stars can be as high as $5 \times 10^{34} \mathrm{erg}$. We conclude that in these prominences there is enough energy to explain the low and medium energetic flares but probably not the very energetic ones.

Acknowledgments. The author thanks Nick Cartledge and Moira Jardine for the careful reading of the manuscript and PPARC for financial support.

\section{References}

Anzer, U. 1969 Solar Phys., 8, 37

Berstein, I. B. et al. 1958, Proc. Roy. Soc., A244, 17

Collier-Cameron, A. and 7 other authors 1990, MNRAS, 247, 415

Lepeltier, T. and Aly, J. J. 1996, A\&A, 306, 645

Spruit, H. C., and Taam, E. R. 1990, A\&A, 229, 475 